\title{
The global burden of pancreatic cancer
}

Giuseppe Lippi ${ }^{1}$, Camilla Mattiuzzi ${ }^{2}$

\begin{abstract}
${ }^{1}$ Section of Clinical Biochemistry, University of Verona, Verona, Italy
${ }^{2}$ Service of Clinical Governance, Provincial Agency for Social and Sanitary Services, Trento, Italy
\end{abstract}

Submitted: 6 August 2019

Accepted: 14 September 2019

Arch Med Sci 2020; 16 (4): 820-824

DOI: https://doi.org/10.5114/aoms.2020.94845

Copyright $\odot 2020$ Termedia \& Banach

\section{Abstract}

Introduction: We provide here an overview on current worldwide epidemiology of pancreatic malignancies, obtained from Global Health Data Exchange (GHDx) and World Health Organization (WHO) repositories.

Material and methods: Electronic searches for collecting information on pancreatic cancer epidemiology were performed in official repositories of GHDx and WHO, and retrieved data were then analyzed.

Results: Overall, 447665 new cases of pancreatic cancer were recorded around the world in 2017 (58.6 per million), with global prevalence of 49.8 per million and 441083 deaths (57.7 per million). Incidence, prevalence and mortality increased by $55 \%, 63 \%$ and $53 \%$ during the last 25 years. Pancreatic cancer accounts for $1.8 \%$ of all cancers, causing $3.9 \%$ of all cancer disabilityadjusted life years (DALYs) and $4.6 \%$ of all cancer deaths. No sex differences can be observed for incidence, prevalence and mortality, whilst DALYs are marginally higher in men. Incidence, prevalence and mortality follow a similar age-related trend, with gradual escalation after 30 years of age, reaching the highest burden after 80 years of age. The DALYs peak between 55 and 74 years, and then decline. The epidemiologic burden is positively associated with socio-demographic status. The largest burden of pancreatic cancers is observed in the East and Asia Pacific region, whilst the lowest is found in the Middle East and North Africa. Pancreatic cancer deaths are expected to increase by $\sim 1.97$-fold by the year 2060 .

Conclusions: Although pancreatic cancer remains relatively infrequent, its clinical, societal and economic burden is noteworthy. Future projections suggest that its burden may double during the next 40 years.

Key words: pancreatic cancer, epidemiology, prevalence, mortality.

\section{Introduction}

The generic term pancreatic cancer includes a class of malignancies originating from the exocrine or endocrine tissue of the pancreas. Pancreatic adenocarcinoma is the most common form, representing approximately $85 \%$ of cases, followed by other less frequent types of exocrine cancers such as squamous cell carcinomas, adenosquamous carcinomas, signet ring cell carcinomas, undifferentiated carcinomas and undifferentiated carcinomas with giant cells [1]. Pancreatic neuroendocrine tumors (PanNET) develop instead from the endocrine tissue of the organ, and usually represent $<5 \%$ of all pancreatic malignancies [2].

Pancreatic cancer is still considered one of the most lethal malignancies, since it remains silent (i.e., does not cause suggestive signs or

\author{
Corresponding author: \\ Prof. Giuseppe Lippi \\ Section of Clinical \\ Biochemistry \\ University Hospital of Verona \\ Piazzale LA Scuro 10 \\ 37134 Verona, Italy \\ Phone: +390458124308 \\ Fax: +39045 8122970 \\ E-mail: giuseppe.lippi@univr.it
}


symptoms) for a rather long time. Then, when patients become symptomatic, the cancer has usually reached an advanced and very frequently incurable stage. According to the American Cancer Society (ACS) [3], the overall 5-year survival rate for pancreatic cancer is dramatically low, approximating $9 \%$. Notably, $97 \%$ of patients with distant cancer (i.e., stage IV) are expected to die after 5 years from diagnosis, whilst the 5 -year survival rates of those with localized (stage $0, \mathrm{I}$, and IIA) or regional (stage IIB and III) cancers are $\sim 34 \%$ and $\sim 12 \%$, respectively. The most important risk factors for pancreatic cancer include tobacco smoke (nearly $25 \%$ of pancreatic malignancies are probably due to cigarette smoking), obesity (body mass index $\left.(B M I) \geq 30 \mathrm{~km} / \mathrm{m}^{2}\right)$, chemicals exposure (especially to benzene, petrochemicals pesticides and certain dyes), diabetes, pancreatitis, Helicobacter pylori infection, as well as some inherited diseases such as hereditary breast and ovarian cancer (HBOC) syndrome, familial atypical multiple mole melanoma (FAMMM) syndrome, familial pancreatitis, hereditary non-polyposis colorectal cancer (HNPCC) syndrome and Peutz-Jeghers syndrome $[4,5]$.

Since pancreatic cancer prevention and management need to be based on quality information, so that effective healthcare policies can be established, we provide here an overview on current worldwide epidemiology of pancreatic cancer.

\section{Material and methods}

Recent data on pancreatic cancer epidemiology were retrieved from Global Health Data Exchange (GHDx), a large worldwide repository of healthrelated information maintained by the Institute for Health Metrics and Evaluation [6]. The Global Burden of Diseases, Injuries, and Risk Factors Study 2017 (GBD 2017) database encompasses thoughtful analysis of disease burden and mortality of 354 different human pathologies from 195 different countries and territories, between the years 1990 and 2017 [7]. The epidemiologic information originates prevalently from survey and surveillance data, admission and outpatient records, health insurance claims and literature studies, accounting for nearly 69000 scientific sites [7]. These sources can be accessed with interactive citation tools from the GHDx database, where citations for specific GBD components are available. Epidemiologic data are finally estimated with the Bayesian meta-regression method DisMod-MR 2.1.

A specific electronic search was conducted in GHDx for the specific condition ("Cause") "pancreatic cancer" [International Classification of Diseases (ICD) codes C25-C25.9, Z85.07, including all primary or metastatic malignant tumor involving the pancreas]. The query was then complemented by selecting additional epidemiologic variables such as "metric" (i.e., "number"), "measure" (i.e., "incidence”, "prevalence”, "DALYs” AND “deaths"), "year" (i.e., "1992" AND "1997" AND "2002" AND “2007” AND “2012” AND "2017”), “Age” (“Under 20" AND "20 to 24" AND "25 to 29" AND "30 to 34" AND "35 to 39" AND "40 to 44" AND "45 to 49" AND "50 to 54" AND "55 to 59" AND "60 to 64" AND "65 to 69" AND "70 to 74" AND "75 to 79" AND "80 plus"), "sex" (i.e., "male" AND "female") and “Location" ("SDI Regions" OR "WB Regions"). The year 2017 was selected for obtaining the current epidemiologic picture, since it is the last searchable year in the GHDx database. Data on mortality projection were instead obtained from the World Health Organization (WHO) database [8]. The data retrieved from the electronic searches were imported into a Microsoft Excel file (Microsoft, Redmond, WA, US), and then analyzed with Microsoft Excel and MedCalc statistical software (MedCalc Software, Ostend, Belgium).

The study was performed in accordance with the Declaration of Helsinki, under the terms of relevant local legislation. Ethics board approval is unnecessary at the local institution (University of Verona) for articles based on free scientific database searches.

\section{Results}

\section{Global epidemiology}

The burden of pancreatic cancer during the past 25 years is shown in Figure 1. A total of 447665 new cases of pancreatic cancer were recorded around the world in 2017 (58.6 cases per million inhabitants), with global prevalence of 380582 cases (49.8 cases per million inhabitants) and 441083 deaths (57.7 per million inhabitants). Overall, incidence, prevalence and mortality have increased by $55 \%, 63 \%$ and $53 \%$ during the last 25 years. Pancreatic cancer currently accounts

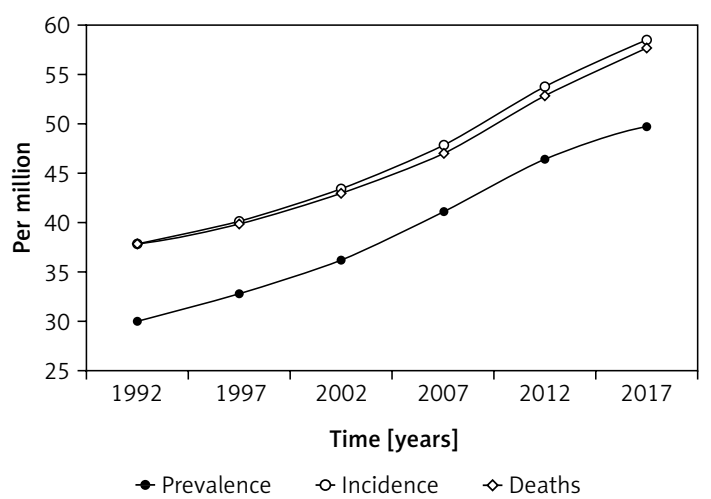

Figure 1. Worldwide incidence, prevalence, causespecific disability-adjusted life years (DALYs) and mortality of pancreatic cancer during the last 25 years 
Table I. Worldwide incidence, prevalence, cause-specific disability-adjusted life years (DALYs) and mortality of pancreatic cancer in the two sexes

\begin{tabular}{|lcccc|}
\hline Sex & Incidence $^{\dagger}$ & Prevalence $^{\dagger}$ & Deaths $^{\dagger}$ & DALYs $^{\dagger}$ \\
\hline Men & 61 & 55 & 59 & 3711 \\
\hline Women & 57 & 45 & 56 & 3542 \\
\hline OR $(95 \% \mathrm{Cl})$ & $1.07(0.75-1.54)$ & $1.22(0.82-1.81)$ & $1.05(0.73-1.52)$ & $1.05(1.00-1.10)$ \\
& $p=0.713$ & $p=0.318$ & $p=0.780$ & $p=0.047$ \\
\hline
\end{tabular}

${ }^{\dagger}$ Per million.

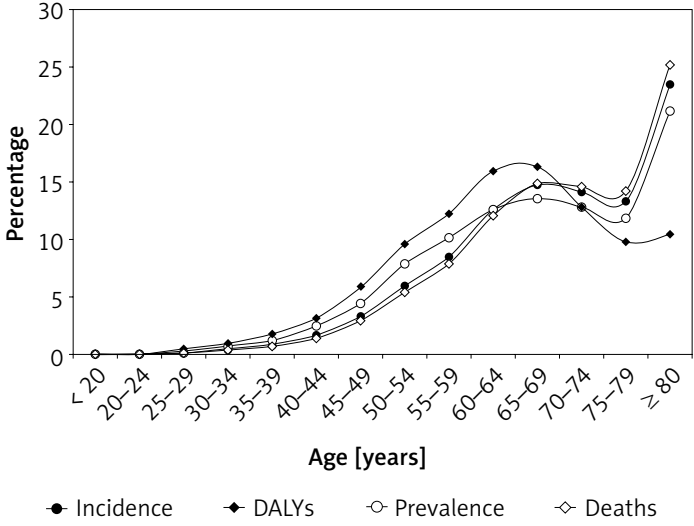

Figure 2. Worldwide incidence, prevalence, causespecific disability-adjusted life years (DALYs) and mortality of pancreatic cancer in different age ranges

for $0.79 \%$ of all-cause global mortality, with a 1.80 -fold increase having occurred during the last 25 years (was $0.44 \%$ in 1992). Among all malignancies, pancreatic cancer is the $14^{\text {th }}$ for incidence ( $1.8 \%$ of all cancers), the $27^{\text {th }}$ for prevalence ( $0.4 \%$ of all cancers), but is the $9^{\text {th }}$ cause of DALYs (3.9\% of all cancers) and the $6^{\text {th }}$ cause of death (4.6\% of all cancers). According to the GBD 2017 Disease and Injury Incidence and Prevalence Collaborators, 66\% pancreatic cancer patients are in primary therapy or the controlled phase, $24 \%$ are in the metastatic stage, whilst $10 \%$ are in the terminal stage [7]. The DALYs for pancreatic cancer have also constantly increased during the last 25 years, from 4.64 million ( $0.18 \%$ of all DALYs) in 1992 to 9.08 million in 2017 (0.36\% of all DALYs; 2-fold increase).

\section{Gender and age epidemiology}

The worldwide sex distribution of pancreatic cancer is shown in Table I. Basically, no statistically significant differences can be observed in terms of incidence, prevalence and mortality, whilst the DALYs value is higher in men than in women with borderline statistical significance $(+4.8 \%$, $p=0.047$ ).

The age distribution of pancreatic cancer in each age range is shown in Figure 2. Incidence, prevalence and mortality follow a similar trend, characterized by a gradual escalation after the age of 30 years, reaching the highest burden in subjects aged 80 years or older ( $>20 \%$ of cases for all these three epidemiologic measures). The age-related DALYs seem to follow a different trend, with a peak between 55 and 74 years ( $58 \%$ of all DALYs for pancreatic cancer), and then declining afterwards.

\section{Socio-demographic epidemiology}

The incidence, prevalence, DALYs and mortality of pancreatic cancer in the different socio-demographic areas are shown in Table II. All epidemiologic measures gradually increase in parallel with SDI, and a highly significant exponential correlation can also be found between SDI rank and incidence $(r=0.993 ; p<0.01)$, prevalence $(r=0.986$; $p<0.01)$, DALYs $(r=0.999 ; p<0.01)$ and mortality ( $r=0.996 ; p<0.01)$. The incidence, prevalence, DALYs and mortality of pancreatic cancer in the different worldwide regions are shown in Figure 3. The trend of all these four epidemiologic measures is extensively overlapping, with a higher burden of pancreatic cancer recorded in the East and Asia

Table II. Incidence, prevalence, cause-specific disability-adjusted life years (DALYs) and mortality of pancreatic cancer in areas with different socio-demographic index (SDI) values

\begin{tabular}{|c|c|c|c|c|}
\hline SDI & Incidence $^{\dagger}$ & Prevalence $^{\dagger}$ & Deaths $^{\dagger}$ & $\operatorname{DALYs}^{\dagger}$ \\
\hline Low & 13 & 10 & 13 & 753 \\
\hline Low-middle & 21 & 16 & 22 & 1424 \\
\hline Middle & 37 & 27 & 39 & 3160 \\
\hline High-middle & 75 & 59 & 77 & 5988 \\
\hline High & 185 & 176 & 172 & 11012 \\
\hline
\end{tabular}




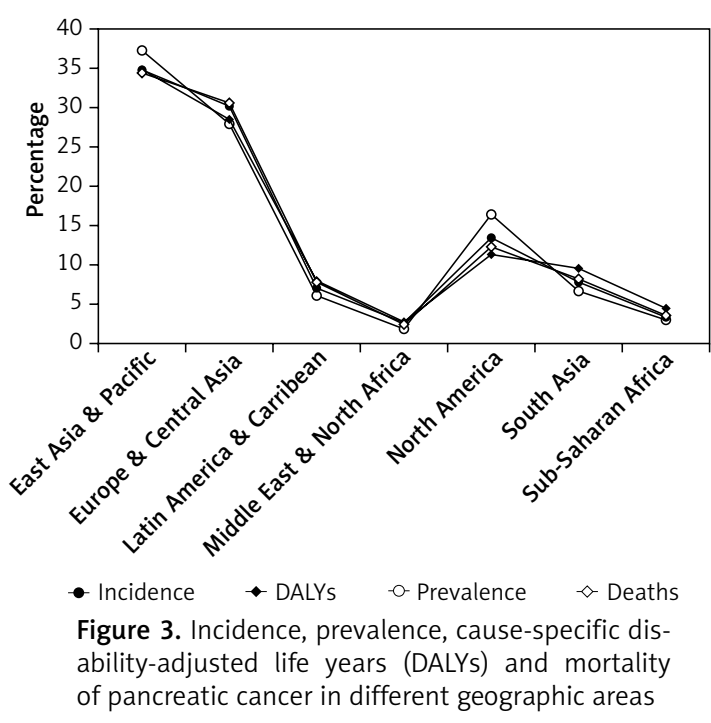

Pacific region (34.5-37.2\% of all cases), followed by Europe and Central Asia (27.9-30.5\% of all cases), whilst the lowest burden is found in Middle East and North Africa (always < $5 \%$ of all cases).

\section{Future projections}

The projection of global mortality for the years 2016-2060 according to WHO data is finally shown in Figure 4. Notably, all-cause mortality will probably rise by $\sim 1.80$-fold during the next 40 years but, even more importantly, all-cancer and pancreatic cancer deaths are expected to both exhibit a larger increase, of approximately 2.08- and 1.97-fold, respectively. The total number of pancreatic cancer deaths will hence likely exceed 730000 in 2060, exhibiting a virtually linear increase during the next 40 years $(r=0.996 ; p=0.004)$.

\section{Discussion}

The results of this first combined analysis based on GHDx [6] and WHO [7] databases, which are the two most comprehensive and reliable healthcare data repositories around the world, attest that pancreatic cancer is still a remarkable cause of clinical, social and economic burden worldwide, and that its burden seems more frequently associated with some specific epidemiologic variables.

The current statistics, and especially WHO projections, demonstrate that the burden of this malignancy is expected to dramatically increase in the next 40 years, following a nearly linear relationship. This will ultimately lead to double pancreatic cancer mortality by the year 2060, approximating a death rate of $>72$ cases per million. Unlike previous reports showing a slightly higher burden of pancreatic cancer in the male sex [9], we found that sex differences are seemingly minimal and mostly not statistically sig-

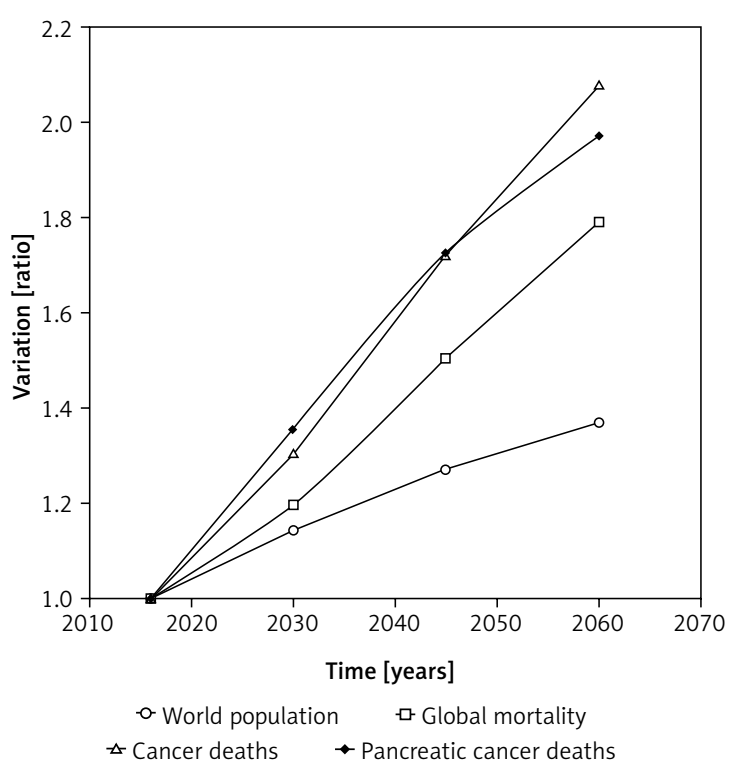

Figure 4. Future trends of mortality for all causes, all types of cancers and pancreatic cancers

nificant (Table I). A marginally higher value of DALYs was found in men compared to women, but the overall difference was modest (+4.8\%). We also originally demonstrated that the geographical distribution of pancreatic cancer presents some paradigmatic trends, displaying especially a strong and positive relationship with the socio-demographic status. All the epidemiologic indices were strongly correlated with the SDI rank (correlations always > 0.98 and $p$-values always $<0.01)$, thus suggesting that preventive and diagnostic strategies will be more effectively implemented in countries with medium to high socio-demographic status. Notably, prevalence and mortality in middle-to-high SDI countries were $\sim 9$-fold (odds ratio $(\mathrm{OR})=8.6$; 95\% confidence interval $(95 \% \mathrm{Cl})$ : 4.8-15.3; $p<0.001)$ and $\sim 7$-fold (OR $=6.7 ; 95 \% \mathrm{Cl}: 4.1-$ $10.9 ; p<0.001)$ higher than in middle-to-low SDI countries, respectively. A larger burden of pancreatic malignancies could also be observed in East Asia and Pacific, as well as in Europe and Central Asia regions, whilst the epidemiologic impact in Middle East and North Africa remained extremely modest. Overall, the prevalence and death rate of pancreatic cancer in the East Asia and Pacific region were $\sim 28$-fold $(\mathrm{OR}=27.6$; $95 \% \mathrm{Cl}$ : $17.6-$ 43.3; $p<0.001)$ and $\sim 20$-fold $(\mathrm{OR}=20.5 ; 95 \% \mathrm{Cl}$ : 13.5-31.2; $p<0.001$ ) higher than in Middle East and North Africa, respectively.

Although our analysis of age distribution confirms previous evidence obtained from different sources and showing that the epidemiologic burden gradually increases with ageing (and displays a modest inflection before the age of 80 years) [9], we originally observed that the DALYs 
value is more accentuated in the age range of $55-74$ years ( $~ 58 \%$ of all DALYs for pancreatic cancer), then gradually declines and stabilizes in very elderly persons (i.e., in those aged 80 years or older) (Figure 2). Since DALYs are a well-known societal measure of disability hindrance in the general population, this evidence would actually mean that pancreatic cancer seemingly poses the largest healthcare and societal burden in middle age people. The exact reason why the DALYs value declines after the age of 65 years remains unclear, although evidence has been provided that the prognosis of this malignancy is consistently worse in the elderly, due to the presence of many comorbidities, organ dysfunctions and the lack of specific treatments in older patients [10].

In conclusion, disease epidemiology is a mainstay for establishing appropriate and timely healthcare interventions aimed at limiting the burden and mortality of human pathologies [11, 12]. According to our analysis, the current worldwide epidemiology of pancreatic cancer highlights an alarming scenario. Although pancreatic cancer remains a relatively rare malignancy $(1.8 \%$ of all cancers), its clinical, societal and economic burden is noteworthy (3.9\% of all DALYs for cancers), and its mortality rate is even higher (4.6\% of all cancers). Future projection attests that the current burden may even double in the next 40 years. Therefore, healthcare policies especially focused on prevention and early diagnosis of pancreatic cancer should be reinforced or redefined, as suggested elsewhere [13]. Additional investments should then be allocated for identifying early diagnostic, prognostic and recurrence biomarkers (e.g., liquid biopsy) [14], as well as for developing more effective therapies aimed at improving both the volume and quality of life in patients with this highly lethal malignancy [15].

\section{Conflict of interest}

The authors declare no conflict of interest.

\section{References}

1. Haeberle L, Esposito I. Pathology of pancreatic cancer. Transl Gastroenterol Hepatol 2019; 4: 50.

2. Salaria SN, Shi C. Pancreatic neuroendocrine tumors. Surg Pathol Clin 2016; 9: 595-617.

3. American Cancer Society. Survival Rates for Pancreatic Cancer. Available at: https://www.cancer.org/cancer/ pancreatic-cancer/detection-diagnosis-staging/survivalrates.html (Accessed: 18.07.2019).

4. McGuigan A, Kelly P, Turkington RC, Jones C, Coleman HG, McCain RS. Pancreatic cancer: a review of clinical diagnosis, epidemiology, treatment and outcomes. World J Gastroenterol 2018; 24: 4846-61.
5. Capasso M, Franceschi M, Rodriguez-Castro Kl, et al. Epidemiology and risk factors of pancreatic cancer. Acta Biomed 2018; 89: 141-6.

6. Institute for Health Metrics and Evaluation. Global Health Data Exchange. Available at: http://ghdx.healthdata.org/gbd-results-tool (Accessed: 18.07.2019).

7. GBD 2017 Disease and Injury Incidence and Prevalence Collaborators. Global, regional, and national incidence, prevalence, and years lived with disability for 354 diseases and injuries for 195 countries and territories, 1990-2017: a systematic analysis for the Global Burden of Disease Study 2017. Lancet 2018; 392: 1789-858.

8. World Health Organization. Projections of mortality and causes of death, 2016 to 2060. Available at: https:// www.who.int/healthinfo/global_burden_disease/projections/en/ (Accessed: 18.07.2019).

9. Rawla P, Sunkara T, Gaduputi V. Epidemiology of pancreatic cancer: global trends, etiology and risk factors. World J Oncol 2019; 10: 10-27.

10. Higuera O, Ghanem I, Nasimi R, Prieto I, Koren L, Feliu J. Management of pancreatic cancer in the elderly. World J Gastroenterol 2016; 22: 764-75.

11. Niklas A, Flotyńska A, Puch-Walczak A, et al. Prevalence, awareness, treatment and control of hypertension in the adult Polish population - Multi-center National Population Health Examination Surveys - WOBASZ studies. Arch Med Sci 2018; 14: 951-61.

12. Söz G, Karakaya Z. The evaluation of geriatric patients who presented with trauma to the emergency department. Arch Med Sci 2019; 15: 1261-8.

13. Cooperman AM, Iskandar ME, Wayne MG, Steele JG. Prevention and early detection of pancreatic cancer. Surg Clin North Am 2018; 98: 1-12.

14. Yadav DK, Bai X, Yadav RK, et al. Liquid biopsy in pancreatic cancer: the beginning of a new era. Oncotarget 2018; 9: 26900-33.

15. O'Hayer KM, Brody JR. Personalized therapy for pancreatic cancer: do we need better targets, arrows, or both? Discov Med 2016; 21: 117-23. 